\title{
99. SOBRE ALGUNOS NEÓFITOS Y OTRAS CITAS INTERESANTES PARA LA FLORA DE ANDALUCÍA OCCIDENTAL
}

Carlos ROMERO ZARCO

On some neophytes and other interesting records for Western Andalusian flora.

Palabras clave. Corología, neófitos, Andalucía, SO de España, florística.

Key words. Chorology, neophytes, Andalucia, SW Spain, floristic.

En las notas que siguen se recogen algunas novedades corológicas que son, en parte, el resultado de investigaciones florísticas del autor en el casco urbano de Sevilla y en las comarcas próximas para el desarrollo del proyecto Flodhis (Romero Zarco y Roales, 2001). Otros materiales fueron amablemente donados por sus recolectores respectivos e incluídos en el Herbario de la Universidad de Sevilla (SEV).

Aira caryophyllea subsp. uniaristata (Lag. \& Rodr.) Maire

HUELVA: El Abalario, 30-III-95, García Murillo et al. (SEV s/n). Hinojos, Coto del Rey, 19-V-84, Devesa et Talavera (SEV 213916).

Novedad para la flora del Parque Natural de Doñana. Recientemente Garrido et al. (2002) señalaron la presencia de esta especie en 7 bosques-isla de la provincia de Huelva, sin indicar localidad alguna, por lo que se considera pertinente publicar algœn testimonio que confirme su presencia en la flora de Doñana. Más datos sobre la distribución de este taxón en las provincias de Huelva y Sevilla pueden encontrarse en Pérez Latorre et al. (2002), quienes lo destacan como novedad para las comarcas que abarca la cuenca del Guadiamar, pero la citan expresamente sólo en La Puebla del Río (Sevilla, pág. 203, tab. 5), y en Santa-Bárbara et al. (2003), quienes la citan en la comarca del Andévalo (Huelva).

\section{Alopecurus myosuroides Hudson}

SEVILLA: Entre Bormujos y Bollullos de la Mitación, urbanización "El Rocío Chico", carril oeste, herbazales ruderales, 15-V-00, C. Romero (SEV 213917). Sevilla, alcorques de la Avenida de La Raza, 15-III96, Talavera (SEV 213918)

Novedad para Andalucía. Especie de distribución paleártica, introducida en Norteamérica y Nueva Zelanda. Segœn el material reseñado por Paunero (1952) esta especie se distribuye en España por el Centro, Norte y Este de la Península y en la Isla de Cabrera. En principio se consideró especie adventicia para la flora andaluza, ya que se encontró por primera vez en un solo lugar transitado por camiones en la zona portuaria, sin embargo su presencia en otros hábitats ruderales lejos de la ciudad parece indicar que está naturalizada y en expansión.

\section{Avena strigosa Schreber subsp. strigosa}

HUELVA: Paimogo, en pastizales próximos a la carretera, 21-III-04, S. Román et al. (SEV 213920); ídem, en campos de cultivos cercanos al pueblo, 20-III-2004, S. Román (SEV 213921).

Novedad para la comarca del Andévalo. Especie de distribución occidental en la 
Península, conocida de Galicia, Portugal y litoral atlántico andaluz (Romero Zarco, 1996).

\section{Bupleurum rigidum subsp. paniculatum} (Brot.) H. Wolff.

CÁDIZ: Jerez de la Frontera, Montes Propios de Jerez, Dehesa de Montifarti, matorral bajo en suelo básico, 25-VI-88, $K$. Schneider (SEV 213933).

Novedad para la Comarca de Algeciras y para el Parque Natural de los Alcornocales. Su área conocida en Andalucía occidental comprende las comarcas de Marisma, Litoral gaditano, Grazalema y Sierras Subbéticas (García Martín, 1987), a las que hay que añadir la cita de Ruiz de Clavijo et al. (1984) para la Campiña Alta sevillana, y la más reciente de Aparicio et al. (2001) para la Campiña Alta gaditana.

\section{Chamaesyce maculata (L.) Small}

HUELVA: Huelva, marisma, terrenos de la Fábrica, 16-VII-82, P. Weickert (SEV 213922).

Novedad para la flora de la provincia de Huelva. Del Sur se ha citado en Sevilla (Valdés, 1987) y en Cádiz (Benedí, 1997; Sánchez García y Galán de Mera, 1997).

\section{Cyperus laevigatus subsp. distachyos (All.)} Maire \& Weiller

SEVILLA: Sevilla, parque del Alamillo, pastizal al borde de una laguna, 22-IV-04, C. Romero (SEV 213923).

Novedad para la Vega sevillana. En Andalucía occidental se ha citado en las comarcas de: Litoral, Marisma, Campiña Alta sevillana (Aparicio y García Martín, 1987), Campiña Alta cordobesa y Subbética cordobesa (Jiménez y Ruiz de Clavijo, 1990).

Eleusine indica (L.) Gaertn. subsp. indica CÁDIZ: Sanlœcar de Barrameda, jardines abandonados del Palacio de
Montpensier, 13-VIII-86, C. Romero (SEV 213924). SEVILLA: Sevilla, subespontánea en jardines, 6-IX-87, G. Labrador (SEV 213925); ídem, alrededores del parque de María Luisa, V-97, C. Romero (SEV 213926).

Al parecer son las primeras citas para ambas provincias. En Andalucía se conocía su presencia en las provincias de Almería (Carretero, 1985), Córdoba (Pujadas Salva y Hernández Bermejo, 1986) y Jaén (Lara Ruiz, 1993), esta última dudosa dada la falta de seriedad del autor de la cita.

\section{Glyceria declinata Bréb.}

CÓRDOBA: Comarca de los pedroches, Villanueva de Córdoba, orillas del arroyo Navabroca, 1-VIII-76, Devesa (SEV 213927).

Novedad para la comarca de los Pedroches segœn la distribución que indica Talavera (1987).

Leptochloa fusca (L.) Kunth subsp. uninervia (J. Presl) N. Snow

CÓRDOBA: Palma del Río, cultivos junto al pueblo en la margen izquierda del Guadalquivir, 9-VI-87, C. López y $C$. Romero (SEV 213928). HUELVA: entre Villablanca y San Silvestre de Guzmán, cuneta junto a un cultivo de naranjos, 12IX-01, L. Fernández Carrillo (SEV 213929). SEVILLA: Isla Mayor, junto al pueblo, 16VII-90, recolector desconocido (SEV 213930). La Puebla del Río, marismas del Guadalquivir, arrozales de la Finca Reina Victoria, 11-VII-2003, F. Montes et al. (SEV 203357; 203359). Entre Lora del Río y La Campana, borde de una acequia, 22-V-98, $M$. García García y M.A. Mateos (SEV 213931). San Juan de Aznalfarache, orillas del Guadalquivir junto a la embasadora de arroz, 2-IX-99, P. Moreno Martínez (SEV 213932).

Novedad para las provincias de Córdoba y de Huelva. Se trata de un neófito de origen 
neotropical potencialmente peligroso como planta invasora de cultivos (Snow and Simon, 1999). El pliego de Palma del Río es el testimonio más antiguo que conocemos de la introducción de esta especie en España. Al parecer fue citada por primera vez para el continente europeo por Peinado et al. (1990, sub Diplachne uninervia), quienes la encontraron también en la comarca de la Vega sevillana a orillas del Guadalquivir (Lora del Río, 18-IX-88). Otras localidades de la Marisma y la Vega sevillanas han sido publicadas por Roales (1997), siempre en cultivos de regadío. Su introducción debe ser reciente y ha invadido de forma especial los campos de arroz. Ha sido citada en cultivos de regadío de Cataluña (Recasens et Conesa, 1995). También se ha introducido recientemente en Israel (Danin et Scholz, 1999) y en Australia, donde se conoce desde 1992 (según Snow et Simon, 1999).

Otro taxón muy similar, Diplachne fascicularis (Lam.) Beauv., se ha introducido recientemente en Extremadura y Andalucía, habiendose citado en cultivos de regadío de la provincia de Badajoz (Devesa, 1991) y bordes de marisma en Huelva (Sánchez Gullón y Rubio García, 1999). Según Snow (1998), tanto Diplachne uninervia como D. fascicularis pertenecen al complejo de Leptochloa fusca (L.) Kunth.

\section{Ophrys dyris Maire}

CÁDIZ: Carretera Ubrique-Jimena de la Frontera, Km 39, 16-III-88, L. Velasco (SEV 213934).

Al parecer se trata del segundo testimonio publicado para la provincia de Cádiz y de un nuevo registro para la lista de endemismos ibero-mauritánicos del Parque Natural de los Alcornocales. La descripción de esta especie, junto con una excelente fotografía, puede verse en el libro sobre las orquídeas de Grazalema (Velasco, 1988), sin embargo la localidad que incluye en el mapa se corresponde con la citada. No obstante la especie también está presente en el Parque Natural de Grazalema, donde Becerra (2003) la señala como novedad para el Parque (en la parte malagueña, Sierra de Líbar) sin sospechar que mucho antes la habían citado de la parte gaditana (Grazalema, 1000 m, 5VI-71, A. et Ch. Nieschalk, según Arnold, 1981, sub O. omegaifera Fleischm.). En Andalucía occidental es especie infrecuente de las comarcas de Sierra Morena (Silvestre, 1987).

\section{Salix alba L.}

SEVILLA: Entre Écija y Herrera, orilla izquierda del río Genil, estación de bombeo del Cortijo la Tambora de San Francisco, 3IV-86, C. López y C. Romero (SEV 213935).

Novedad para la Campiña Baja. Silvestre (1987) la citó en las comarcas de la Vega del Guadalquivir, Condado-Aljarafe, Litoral y Grazalema, y Muñoz en la Subbética cordobesa, según recogen García Montoya et Muñoz (1990).

\section{Solanum cornutum Lam.}

SEVILLA: Sevilla, zona portuaria, avda. Reina Mercedes, 8-VII-86, C. Romero (SEV 118383).

Especie nativa de México y SW de los Estados Unidos que se encuentra localmente naturalizada en países del centro y Sur de Europa (Hawkes \& Edmonds, 1972). La primera mención de su presencia en España debe ser la de Valdés (1987) quien la citó de una forma un tanto irregular en la Flora Vascular de Andalucía occidental, donde aparece comentada sin autor ni referencia alguna y en letra pequeña, bajo la descripción del género, sin que fuera incluída en la clave de especies ni en el índice. El testimonio de esa primera aparición es el que damos arriba. Recolectada en un solar urbano recientemente urbanizado. Posteriormente fue encontrada en las cercanías de Badajoz 
(Ruiz Téllez, 1995), pero aquí no ha vuelto a verse.

Sporobolus indicus (L.) R. Br.

CÁDIZ: Puerto de Santa María, camping, 28-VIII-86, C. Romero (SEV 213936). SEVILLA: Sevilla, alrededores del Parque de María Luisa, 11-VII-91, C. Infante (SEV 213937).

Al parecer es la primera vez que se cita esta especie en Andalucía. Que sepamos, en España se ha citado de Castellón (Roselló y Peris, 1983), Extremadura (Viera, 1991), Madrid (Gavilán et al., 1993), y en provincias situadas más al Norte, en Aragón (Sanz Elorza, 1997), Castilla León (Llamas y Acedo, 1997; Navarro y García Río, 1990), Cataluña (Sennen, 1916, entre otras referencias), Galicia (Valdés Bermejo et al., 1995) y País Vasco (numerosas citas). Aquí se comporta de momento como especie adventicia escapada de cultivo. La referencia más antigua debe ser la de Sennen (1.c.), quien la citó a orillas del Muga (Gerona) y del Besós (Barcelona).

\section{Vicia hirsuta (L.) S.F. Gray}

SEVILLA: Sevilla, Isla de la Cartuja, en zonas verdes abandonadas de los terrenos de la Expo-92, 22-IV-2004, C. Romero (SEV 213938).

Novedad provincial. Especie frecuente en zonas montañosas de la Península y Baleares (Romero Zarco, 1999) pero muy poco conocida en Andalucía occidental, donde œnicamente había sido citada hasta la fecha en la Serranía de Grazalema (Aparicio y Romero Zarco, 1986). Se trata probablemente de una población adventicia de reciente introducción.

\section{Zoysia matrella (L.) Merr.}

HUELVA: Palos de la Frontera, La Rábida, adventicia en césped de Pennisetum clandestinum, 10-V-2000, J. Macías y E.
Sánchez Gullón (SEV 213939).

Gramínea de la tribu Cynodonteae, originaria de las costas del Océano êndico y del Mar de China, actualmente cultivada en céspedes y praderas artificiales en América $\mathrm{y}$ en diversas regiones tropicales y subtropicales. Son varias las especies del género que se han utilizado bajo el nombre comercial de zoysiagrass, y numerosos los híbridos y cultivares seleccionados en Norteamérica, pero Z. matrella es la única señalada como hierba invasora por varios autores, entre ellos Häfliger and Scholz (1981). Su presencia en Huelva ha sido reseñada por D. Enrique Sánchez Gullón, que la identificó como Z. tenuifolia Willd. ex Thiel. Zanjamos la disparidad de criterio otorgando a $Z$. matrella su sentido más amplio, lo que deja a $Z$. tenuifolia en la sinonimia. Que sepamos es la primera vez que se encuentra como especie adventicia en Europa, aunque aquí, de momento, aún no ha colonizado cultivos agrícolas ni hábitats ruderales. Las semillas de Pennisetum clandestinum, especie conocida comercialmente como «hierba kikuyu», se venden en establecimientos de jardinería. Es posible que la contaminación ocasional de estos lotes de semillas alóctonas sea la causa de la introducción fortuita de especies potencialmente invasoras.

AGRADECIMIENTOS. Agradezco al Profesor Luis Velasco (Ubrique) y a D. Enrique Sánchez Gullón (Huelva) el envío de los materiales de los géneros Ophrys y Zoysia respectivamente y al Dr. Francisco Javier Salgueiro (Franja), Conservador del Herbario de la Universidad de Sevilla, la bœsqueda de material en el herbario.

\section{BIBLIOGRAFÍA}

APARICIO, A. y F. GARCÍA MARTÍN -1987Cyperus L., in: B. VALDÉS, S. TALAVERA y E.F. GALIANO (eds.), Flora Vascular de Andalucía occidental 1: 238-243. Ketres 
Editora S.A., Barcelona.

APARICIO, A., C. PÉREZ PORRAS y G. CEBALLOS -2001- Inventario $y$ caracterización florística de los 'Bosquesisla' de la campiña de Cádiz. Consejería de Medio Ambiente, Junta de Andalucía.

APARICIO, A. y C. ROMERO ZARCO -1986Notas taxonómicas y corológicas sobre la flora de Andalucía occidental 188. Vicia hirsuta, Lagascalia 14(1): 142.

ARNOLD, J.E. (1981) Notas para una revisión del género Ophrys L. (Orchidaceae) en Cataluña. Collect. Bot. (Barcelona) 12: 5-45.

BECERRA PARRA, M. -2003-Contribuciones a la flora vascular de Andalucía (España). 91. Nuevas aportaciones al conocimiento de la flora del macizo de Líbar (Cádiz-Málaga). II. Acta Bot. Malacitana 2: 247-251.

BENEDÍ, C. -1997- Chamaesyce Gray, in: S. CASTROVIEJO et al. (eds.), Flora Iberica 7: 286-297. CSIC, Madrid.

CARRETERO, J.L. -1985-Aportaciones a la flora exótica valenciana. Collect. Bot. (Barcelona) 16: 133-136.

DANIN, A. y H. SCHOLZ -1999- Leptochloa uninervia, in: W. GREUTER y T. RAUS (eds.), Med-Checklist Notulae 18, Wildenowia 29: 66.

DEVESA, J. A. -1991-Diplachne Beauv., in: J.A. DEVESA (ed.), Las Gramineas de Extremadura 260-262. Universidad de Extremadura.

GARCÍA MARTÍN, F. -1987- Bupleurum L., in: B. VALDÉS, S. TALAVERA y E.F. GALIANO (eds.), Flora Vascular de Andalucia occidental 2: 308-314. Ketres Editores S.A., Barcelona.

GARCÍA MONTOYA, F. y J. MUÑOZ -1990Notas taxonómicas y corológicas para la flora vascular de Andalucía y del Rif. 18. Novedades corológicas para la flora de Andalucía occidental. Lagascalia 16(1): 146168

GARRIDO, B., A. APARICIO, C. PÉREZ PORRAS, J. APARICIO, F. GRACÍA MARTÍN, L. FERNÁNDEZ CARRILLO y M.A. CARRASCO -2002- Contribuciones a la flora vascular de Andalucía (España). 80. Flora de Interés en bosques-isla de Andalucía occidental. Acta Bot. Malacitana 27: 295-308.
GAVILÁN, R., J.E. ECHEVERRÍA y I. CASAS -1993- Catálogo de la flora vascular de la Ciudad Universitaria de Madrid (España). Bot. Complut. 18: 175-201.

HAWKES, J.G. y J.M. EDMONDS -1972Solanum L., in: T.G. TUTIN et al. (eds.) Flora Europaea 3: 197-199. Cambridge University Press.

JIMÉNEZ, M. y E. RUIZ DE CLAVIJO -1990Notas taxonómicas y corológicas para la flora vascular de Andalucía y del Rif. 16. Nuevas áreas para la flora de Andalucía occidental. Lagascalia 16(1): 132-145.

LARA RUIZ, J. -1993- Contribución al conocimiento de la flora de Andalucía oriental. I. Lagascalia 17(1): 105-118.

LLAMAS, F. y C. ACEDO -1997- De plantis legionensibus. Notula XV. Studia Bot. 16: 139-141.

NAVARRO, F. y R. GARCÍA RÍO -1990Fragmenta chorologica occidentalia 23322341. Anales Jard. Bot. Madrid 47(2): 219220.

PAUNERO, E. -1952- Las especies españolas del género Alopecurus. Anales Jard. Bot. Madrid 10(2): 301-345.

PEINADO, M, J.M. MARTÍNEZ PARRA, C. BARTOLOMÉ, J. ÁlVAREZ y M. DE LA CRUZ -1990- Notas taxonómicas y corológicas para la flora vascular de Andalucía y del Rif. 13. Nuevas plantas y nuevas áreas para la flora de Andalucía occidental. Lagascalia 16(1): 125-129.

PÉREZ LATORRE, A.V., P. NAVAS, D. NAVAS, Y. GIL y B. CABEZUDO -2002- Datos sobre la flora y vegetación de la cuenca del río Guadiamar (Sevilla-Huelva, España). Acta Bot. Malacitana 27: 189-228.

PUJADAS SALVA, A. y J.E. HERNÁNDEZ BERMEJO -1986- Contribución al conocimiento de la flora arvense y ruderal de la provincia de Córdoba. Lagascalia 14: 203225.

RECASENS, J. y J. A. CONESA -1995- Nuevas malas hierbas alóctonas en los cultivos de regadío de Cataluña. Actas del Congreso de la Sociedad Española de Malherbología 1995, 59-65.

ROALES, J. -1997- Contribución al conocimiento de la flora de Sevilla. I: 
novedades corológicas para la provincia. Lagascalia 20(1): 129-149.

ROMERO ZARCO, C. -1996- Sinópsis del género Avena L. (Poaceae, Aveneae) en España peninsular y Baleares. Lagascalia 18(2): 171-198.

ROMERO ZARCO, C. -1999- Vicia L., in: S. TALAVERA, C. AEDO, S. CASTROVIEJO, A. HERRERO, C. ROMERO ZARCO, F.J. SALGUEIRO y M. VELAYOS (eds.), Flora Iberica VII (I). Leguminosae (partim): 360417. CSIC, Madrid.

ROMERO ZARCO, C. y J. ROALES -2001Flodhis 2001, Flora Didáctica Hispalense, CD-Rom. Secretariado de Recursos Audiovisuales y Nuevas Tecnologías de la Universidad de Sevilla.

ROSELLÓ, R. y J.B. PERIS -1983-Algunos neófitos de la provincia de Castellón. Fontqueria 28: 53-56.

RUIZ DE CLAVIJO, E., B. CABEZUDO y E. DOMÍNGUEZ -1984- Contribución al estudio florístico de las serranías subbéticas de la provincia de Sevilla. Acta Bot. Malacitana 9: 169-232.

RUIZ TÉLLEZ, T. -1995- Solanaceae, in: J.A. DEVESA, Vegetación y Flora de Extremadura: 442-444. Universidad de Extremadura.

SÁNCHEZ GARCÍA, I. y A. GALÁN DE MERA -1997- Fragmenta chorologica occidentalia, 5927-5933. Anales Jard. Bot. Madrid 55(1): 152.

SÁNCHEZ GULLÓN, E. y J.C. RUBIO GARCÍA -1999- Novedades florísticas para el litoral de Huelva y provincia. I. Lagascalia 21(1): 7-16.

SANTA-BÁRBARA, C. y B. VALDÉS -1997Novedades florísticas para la provincia de Huelva. I. Lagascalia 20(1): 151-160.

SANTA-BÁRBARA, C., B. VALDÉS y F.J. PINA -2003-Contribuciones a la flora vascular de Andalucía (España). 90. Novedades corológicas para la comarca del Andévalo (provincia de Huelva, SO de España). IV. Acta Bot. Malacitana 28: 237-251.

SANZ ELORZA, M. -1997- Fragmenta chorologica occidentalia 6253-6267. Anales Jard. Bot. Madrid 55(2): 453-454.

SENNEN, F. -1916- Liste des plantes observées aux alentours d'Igualada por D. Ramón Queral Gili. Bol. Soc. Aragonesa Ci. Nat. 15: 94-105.

SILVESTRE, S. -1987- Salicaceae, Orchidaceae, in: B. VALDÉS, S. TALAVERA y E.F. GALIANO (eds.), Flora Vascular de Andalucía occidental 1: 367-372; 3: 495-519. Ketres Editora S.A., Barcelona.

SNOW, N. -1998- Nomenclatural changes in Leptochloa P. Beauvois sensu lato (Poaceae, Chloridoideae). Novon 8: 77-80

SNOW, N. \& B. SIMON -1999- Taxonomic status and Australian distribution of the weedy neotropical grass Leptochloa fusca subsp. uninervia, with an updated key to Australian Leptochloa (Poaceae, Chloridoideae). Austrobaileya 5(2): 299-305.

TALAVERA, S. -1987- Glyceria R. Br., in: B. VALDÉS, S. TALAVERA y E.F. GALIANO (eds.), Flora Vascular de Andalucía occidental 3: 357-359. Ketres Editora S.A., Barcelona.

VALDÉS, B. -1987-Euphorbiaceae, Solanaceae in: B. VALDÉS, S. TALAVERA y E.F. GALIANO (eds.), Flora Vascular de Andalucia occidental 2: 220-241; 351-362. Ketres Editora S.A., Barcelona.

VALDÉS BERMEJO, E., F.J. SILVA PANDO, V. RODRÍGUEZ GRACIA, F. GÓMEZ VIGIDE \& X.R. GARCÍA MARTÍNEZ -1995Aportaciones a la flora de Galicia,V. Nova Acta Cientifica Compostelana (Bioloxía) 5: 135-152.

VELASCO, L. -1988- Orquideas del Parque Natural de Grazalema. Serie Monografías del Medio Ambiente, $\mathrm{n}^{\circ}$ 7, Agencia de Medio Ambiente, Junta de Andalucía.

VIERA, C. -1991-Sporobolus R. Br., in: J.A. DEVESA (ed.), Las Gramineas de Extremadura 260-262. Universidad de Extremadura.

Aceptado para su publicación en octubre de 2004

Dirección del autor. Depto. de Biología Vegetal y Ecología, Universidad de Sevilla, Apdo. 1095, E-41080 Sevilla 